\title{
MRI-guided targeting delivery of doxorubicin with reduction-responsive lipid-polymer hybrid nanoparticles
}

\author{
Bo $\mathrm{Wu}^{1,2}$ \\ Shu-Ting Lu' \\ Kai Deng² \\ Hui Yu ${ }^{2}$ \\ Can Cui ${ }^{2}$ \\ Yang Zhang ${ }^{2}$ \\ Ming $\mathrm{Wu}^{2}$ \\ Ren-Xi Zhuo ${ }^{2}$ \\ Hai-Bo Xu' \\ Shi-Wen Huang ${ }^{2}$ \\ 'Department of Radiology, Zhongnan \\ Hospital of Wuhan University, ${ }^{2}$ Key \\ Laboratory of Biomedical Polymers, \\ Ministry of Education, Department of \\ Chemistry, Wuhan University, Wuhan, \\ People's Republic of China
}

Correspondence: Shi-Wen Huang Key Laboratory of Biomedical Polymers, Ministry of Education, Department of Chemistry, Wuhan University, 299 Bayi Road, Wuhan 430072,

People's Republic of China

Tel/fax +86 2768755317

Email swhuang@whu.edu.cn

Hai-Bo Xu

Department of Radiology, Zhongnan Hospital of Wuhan University, I69 Donghu Road, Wuhan 43007I, People's Republic of China Tel/fax +8627 678I 2533 Email xuhaibol I20@hotmail.com
This article was published in the following Dove Press journal:

International Journal of Nanomedicine

14 September 2017

Number of times this article has been viewed

\begin{abstract}
In recent years, there has been increasing interest in developing a multifunctional nanoscale platform for cancer monitoring and chemotherapy. However, there is still a big challenge for current clinic contrast agents to improve their poor tumor selectivity and response. Herein, we report a new kind of Gd complex and folate-coated redox-sensitive lipid-polymer hybrid nanoparticle (Gd-FLPNP) for tumor-targeted magnetic resonance imaging and therapy. Gd-FLPNPs can simultaneously accomplish diagnostic imaging, and specific targeting and controlled release of doxorubicin (DOX). They exhibit good monodispersity, excellent size stability, and a well-defined core-shell structure. Paramagnetic nanoparticles based on gadolinium-diethylenetriaminepentaacetic acid-bis-cetylamine have paramagnetic properties with an approximately two-fold enhancement in the longitudinal relaxivity compared to clinical used Magnevist. For targeted and reduction-sensitive drug delivery, Gd-FLPNPs released DOX faster and enhanced cell uptake in vitro, and exhibited better antitumor effect both in vitro and in vivo.
\end{abstract}

Keywords: redox-sensitive, tumor-targeted, gadolinium, contrast agents, PLGA

\section{Introduction}

Magnetic resonance imaging (MRI) has long been a powerful tool for efficient diagnosis and therapeutic monitoring of cancers. Paramagnetic gadolinium ions $\left(\mathrm{Gd}^{3+}\right)$ with seven unpaired electrons can efficiently alter the relaxation time of the surrounding water protons. ${ }^{1,2}$ As the major category of MRI contrast agents (CAs) approved for clinical applications, Gd-based complexes such as gadodiamide, gadopentetate dimeglumine, gadoteridol, and gadoterate meglumine are widely used in clinics. In recent years, impressive progress has also been made in the new form and multifunction CA, including micelles, ${ }^{3,4}$ macromolecules, ${ }^{5}$ supramolecular aggregates, ${ }^{6}$ and liposomes, ${ }^{7}$ which have demonstrated good performance in enhancing the MR contrast of early stage tumor. However, there is still a big challenge for current clinic CAs to improve their poor tumor selectivity and response.

Chemotherapy as the most effective treatment modality for cancer is still used as the frontline approach in clinics. One major hindrance in chemotherapy is the systemic distribution of anticancer drugs and concomitant serious side effects. ${ }^{8,9}$ As a solution to this problem, many different effective response drug delivery systems were designed to reduce severe side effects and improve the anticancer effect of anticancer drugs, ${ }^{10,11}$ such as redox-sensitive drug delivery platforms. ${ }^{12-15}$ Redox-sensitive nanocarriers can achieve an annihilating effect and low toxicity on account of the sudden intracellular 
burst of encapsulated drugs caused by the cleavage of disulfide bonds in the redox environment of cancer cells. ${ }^{16-18}$ Our earlier studies have demonstrated the advantages of the amphiphilic reduction-sensitive polymer of monomethoxypoly-(ethylene glycol)-S-S-hexadecyl (mPEG-S-S-C ${ }_{16}$ ) for the effective intracellular delivery of anticancer drugs. ${ }^{13,19}$

To further improve the therapeutic efficiency and reduce side effects, a better strategy was to develop moleculartargeted nanoparticle (NP) therapeutic carriers. Folate receptor (FR), an attractive overexpressed receptor by several human tumors such as ovarian cancer, breast cancer, and epidermoid carcinoma of the oral cavity ${ }^{20-22}$ presented an effective means for selective delivery of therapeutics. Folate, which can bind very firmly to FRs $\left(\mathrm{K}_{\mathrm{d}} \sim 10^{-10} \mathrm{M}\right),{ }^{23}$ has been proved to effectively improve the drug uptake efficiency.

Hence, we developed a kind of folate-targeted, Gd-coated, and redox-sensitive NP that can achieve both imaging and therapy with redox-sensitivity and active targeting ability. Lipid-polymer hybrid nanoparticles (LPNPs), which combine the biosafety of liposomes and versatility of polymeric NPs into a single delivery system, were a good choice for this multifunctional nanocarrier, which uses folate as the targeted ligand and amphiphilic reduction-sensitive polymer to achieve intracellular release, and then improve the therapeutic effect. Compared to traditional nanoplatforms, Gd-coated folate-targeted lipid-polymer hybrid nanoparticles (Gd-FLPNPs) can simultaneously accomplish specific targeting, controlled release of anticancer drugs, MRI, and simultaneous therapy. The Gd-FLPNPs were composed of a biodegradable hydrophobic poly(D,L-lactide-co-glycolide) (PLGA) core and a paramagnetic liposome shell which was based on gadolinium-diethylenetriaminepentaacetic acidbis-tetradecylamide (Gd-DTPA-BC ${ }_{16}$ ), 1,2-distearoyl-snglycero-3-phosphoethanolamine-N-carboxy (polyethylene glycol) 2000-folate (DSPE-PEG ${ }_{2 \mathrm{k}}$-folate), and mPEG-S$\mathrm{S}_{-} \mathrm{C}_{16}$. The PLGA core was employed to load and release hydrophobic drugs. The paramagnetic liposome shell offers many advantages such as folate targeting provided by DSPE$\mathrm{PEG}_{2 \mathrm{k}}$-folate, redox-sensitivity provided by $\mathrm{mPEG}-\mathrm{S}-\mathrm{S}-\mathrm{C}_{16}$, and contrast-enhanced ability provided by Gd-DTPA-BC ${ }_{16}$. The structure of Gd-FLPNPs is shown in Figure 1.

\section{Materials and methods}

\section{Materials}

The synthesis of DTPA-BC ${ }_{16}$ and Gd-DTPA-BC ${ }_{16}$ was carried out by the method of Kimpe et al. ${ }^{24}$ Gd-FLPNPs were prepared using a previously reported single-step assembly method. ${ }^{14}$ For comparison, non-targeted Gd-LPNPs
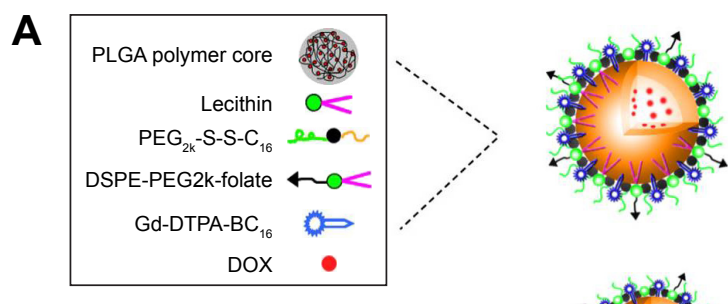

B

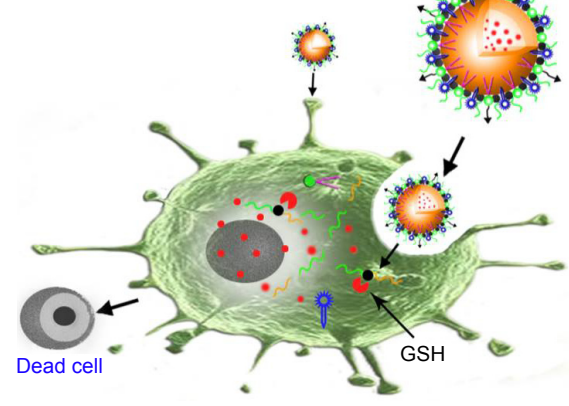

Figure I Schematic illustration of the formation of Gd-FLPNPs and cancer-targeted intracellular drug delivery.

Notes: (A) The amphiphilic mPEG-S-S-C $C_{16}$, contrast agent Gd-DTPA-BC ${ }_{16}$, targeted ligand, and anticancer drug DOX were self-assembled into Gd-FLPNPs. (B) Uptake of Gd-FLPNPs by cancer cells via the active targeting strategy and the anticancer drug was rapidly released for cancer therapy upon the triggering of GSH in cancer cells.

Abbreviations: Gd-FLPNPs, Gd-coated folate-targeted lipid-polymer hybrid nanoparticles; DOX, doxorubicin; GSH, glutathione; mPEG-S-S-C ${ }_{16}$, monomethoxypoly-(ethylene glycol)-S-S-hexadecyl; Gd-DTPA-BC ${ }_{16}$, gadolinium-diethylenetriaminepentaacetic acid-bis-tetradecylamide; PLGA, poly(D,L-lactide-co-glycolide); $\mathrm{DSPE}_{\mathrm{PEG}}$-folate, I,2-distearoyl-sn-glycero-3-phosphoethanolamine-N-carboxy (polyethylene glycol) 2000-folate.

were also prepared using the same above-mentioned method. DSPE-PEG $_{2 \mathrm{k}}$ and DSPE-PEG ${ }_{2 \mathrm{k}}$-folate were purchased from Shanghai Advanced Vehicle Technology Co, Ltd (Shanghai, People's Republic of China). $\mathrm{mPEG}_{2 \mathrm{k}}-\mathrm{S}-\mathrm{S}-\mathrm{C}_{16}$ was synthesized using a method reported in our previous studies. ${ }^{14}$ The consumables of cell culture in this work were purchased from Beijing Dingguo Changsheng Biotechnology Co, Ltd (Beijing, People's Republic of China). PLGA (75:25, molecular weight: 90,000-126,000) and lecithin were purchased from Sigma-Aldrich (St Louis, MO, USA). Diethylenetriaminepentaacetic acid (DTPA), N,N-dimethyl formamide (DMF), DL-dithiothreitol (DTT), triethylamine (TEA), and dimethylsulfoxide (DMSO) were all purchased from Shanghai Chemical Co (Shanghai, People's Republic of China). All solvents used in this study were of highperformance liquid chromatography grade.

\section{Synthesis of ligands}

In brief, DTPA (3.92 g, $10 \mathrm{mmol}$ ) was dissolved in acetic anhydride $(19 \mathrm{~mL}, 200 \mathrm{mmol})$ at $70^{\circ} \mathrm{C}$ for 10 hours under argon atmosphere. The solvent was evaporated under reduced pressure. The obtained crude product of DTPA dianhydride was washed twice with acetic anhydride. Then, DTPA 
dianhydride (714 mg, $2 \mathrm{mmol}$ ) and cetylamine $(968 \mathrm{mg}$, $4 \mathrm{mmol}$ ) were dissolved in anhydrous DMF for 12 hours. After solvent evaporation under reduced pressure, the obtained crude product was purified by preparative thin layer chromatography on silica.

\section{Synthesis of the Gd-DTPA-BC ${ }_{16}$ complexes}

Gd-DTPA-BC ${ }_{16}$ was synthesized by the general procedure as follows: hydrated $\mathrm{GdCl}_{3}$ salt $(1.1 \mathrm{mmol})$ in $\mathrm{H}_{2} \mathrm{O}(1 \mathrm{~mL})$ was added to the ligand (Gd-DTPA-BC $\mathrm{B}_{16}, 1 \mathrm{mmol}$ ) dissolved in pyridine $(30 \mathrm{~mL})$, and the mixture was heated at $70^{\circ} \mathrm{C}$ for 3 hours. The solvents were evaporated under reduced pressure and the crude product was then heated at reflux in ethanol for 1 hour. After the mixture had cooled to room temperature, the complex was filtered off and dried in vacuo. The absence of free gadolinium was checked with xylenol orange indicator.

\section{Preparation of paramagnetic Gd-FLPNPs}

Gd-FLPNPs were prepared using a previously reported single-step assembly method. In brief, prior to the GdFLPNPs preparation, doxorubicin hydrochlorate $(\mathrm{DOX} \cdot \mathrm{HCl})$ was stirred with twice the molar amount of TEA in DMF for 10 hours to obtain lipophilic doxorubicin (DOX). DOX $(5 \mathrm{mg}$ ) was dissolved in $10 \mathrm{~mL}$ of DMF. Then the polymer of PLGA (40 mg) was added to the solution and stirred at room temperature for 2 hours. DSPE-PEG ${ }_{2 \mathrm{k}}$-folate, $\mathrm{mPEG}-\mathrm{S}-\mathrm{S}-\mathrm{C}_{16}$, Gd-DTPA-BC ${ }_{16}$, and lipid lecithin (weight ratio $1 / 6 / 6 / 2$, total weight $30 \mathrm{mg}$ ) were dissolved in $30 \mathrm{~mL}$ of $4 \mathrm{wt} \%$ ethanol aqueous solution at $65^{\circ} \mathrm{C}$, and the DOX/PLGA solution was added dropwise under gentle stirring. Then, the mixed solution was vortexed vigorously for 3 minutes followed by dialyzing against ultrapure water at $25^{\circ} \mathrm{C}$ for 48 hours. Finally, the NPs were concentrated using an Amicon Ultra-4 Centrifugal Filter (EMD Millipore, Billerica, MA, USA; molecular weight cut-off $[\mathrm{MWCO}]=8,000)$. To serve as a control, non-targeted paramagnetic Gd-LPNPs and FLPNPs were also prepared by the above process, and DSPE-PEG ${ }_{2 \mathrm{k}}$-folate or Gd-DTPA-BC ${ }_{16}$ was replaced by DSPE-PEG ${ }_{2 \mathrm{k}}$. Additionally, DOX-free Gd-FLPNPs or DOX-free Gd-LPNPs were also prepared using the above protocol but without DOX.

\section{Physicochemical characterizations of Gd-FLPNPs and Gd-LPNPs}

The particle size and size distribution of the drug loaded NPs were measured by dynamic light scattering (DLS, 90Plus,
Brookhaven Instruments Co, Holtsville, NY, USA). Transmission electron microscopy (TEM, JEM-100CX; JEOL, Tokyo, Japan) was used to observe the micelle morphology at an operating voltage of $100 \mathrm{kV}$. Each result was an average of triplicate measurements. X-ray photoelectron spectroscopy spectra (XPS, Kratos XSAM800 spectrometer; Kratos, Manchester, UK) was employed to investigate the surface chemistry of the NPs, using $\mathrm{Mg} \mathrm{K} \alpha$ radiation $(1,253.6 \mathrm{eV})$ as the exciting source operated at $11.5 \mathrm{kV}$ and $17 \mathrm{~mA}$.

\section{Drug encapsulation efficiency (EE) and responsive drug release}

To evaluate the drug EE and loading efficiency (LE), a predetermined aliquot of Gd-FLPNPs or Gd-LPNPs was collected by freeze drying, and then the dry NPs were dissolved in DMSO. After that, the DOX concentration in DMSO was measured by fluorescence measurement (excitation at $485 \mathrm{~nm}$ ) using a calibration curve constructed from DOX solutions with different DOX concentrations. EE was calculated as (actual amount of drug encapsulated in NPs)/ (initial amount of drug used in the fabrication of NPs) $\times 100 \%$. LE $(\%)=($ amount of drug in particles/amount of the feeding material and drug) $\times 100 \%$.

Two milliliters (DOX, $1 \mathrm{mg} / \mathrm{mL}$ ) of Gd-FLPNPs were transferred to a dialysis tube (MWCO 8,000). Then, it was immersed into a tube containing $10 \mathrm{~mL}$ of phosphate-buffered saline (PBS; $10 \mathrm{mM}, \mathrm{pH} 7.4$ ) with or without $10 \mathrm{mM}$ DTT in a shaker shaking at $120 \mathrm{rpm}$ and $37^{\circ} \mathrm{C}$. At designated time intervals, $5 \mathrm{~mL}$ of the external buffer was replaced with the corresponding fresh buffer solution. Then, DOX quantity was determined by fluorescence measurement (excitation at $485 \mathrm{~nm}$ ) using a calibration curve constructed from DOX solutions with different DOX concentrations. The error bars were obtained from triplicate samples.

\section{Cell culture}

Cervical cancer cell (KB cell) was purchased from the China Center for Type Culture Collection (Wuhan University) and cultured in RPMI 1640 medium, folate-free RPMI 1640 medium, supplemented with $4 \times 10^{-3} \mathrm{M}$ L-glutamine, $10 \%$ fetal bovine serum (FBS), and $1 \%$ antibiotics at $37^{\circ} \mathrm{C}$ in a humidified atmosphere containing $5 \% \mathrm{CO}_{2}$.

\section{Animals and tumor-bearing mice models}

Female BALB/c nude mice were obtained from Beijing HFK Bioscience Co Ltd (Beijing, People's Republic of China) with body weights of 18-20 g and housed under standard pathogen-free conditions $\left(60 \%\right.$ relative humidity, $20^{\circ} \mathrm{C}$ 
room temperature) with a 12 hours light/12 hours dark cycle. Animals were acclimated to this condition for 5 days prior to treatment. These nude mice were subcutaneously inoculated with $\mathrm{KB}$ cells $\left(2 \times 10^{6}\right.$ cells per mice $)$ at backside to establish the subcutaneous transplanted tumor model. Procedures involving animals and their care were conducted in conformity with EU Directive 2010/63/EU for animal experiments and was approved by the Animal Welfare Committee of the Animal Experiment Center of Wuhan University.

\section{In vitro MRI property}

Gd-FLPNPs, Gd-LPNPs, and Magnevist were diluted with deionized water to obtain various concentrations of CA. Two hundred microliters of different concentrations of samples were added to a 96-well plate. The in vitro MRI test was performed with a Siemens Prima 3.0T MRI scanner (Siemens, Munich, Germany). $\mathrm{T}_{1}$-weighted MR images of Gd-FLPNPs, Gd-LPNPs, and a commercially available CA (Magnevist) were obtained under the following experimental conditions: recycle time $700 \mathrm{~ms}$, echo delay time $12 \mathrm{~ms}$. For relaxivity-coefficient measurements, NP samples were prepared with varying $\mathrm{Gd}^{3+}$ concentrations. The mean value of a region of interest was used to determine the relaxivity $\left(r_{1}\right)$. $r_{1}$ which reflected the proton-relaxation-enhancement ability of NPs in water was calculated as follows: $r_{1}=\left(1 / T_{1}-1 / T_{1} d\right) /$ [Gd], where $1 / \mathrm{T}_{1}$ was the longitudinal relaxation rate contrast with the presence of a paramagnetic species, $1 / \mathrm{T}_{1} \mathrm{~d}$ represented longitudinal relaxation rate contrast with the absence of paramagnetic species, and [Gd] represented the concentration of paramagnetic NPs (mM).

\section{In vivo $T_{1}$-weighted $M R I$}

To evaluate the enhancement of Gd-FLPNPs in MRI, C6 cells in the tumor-bearing mouse were scanned by a Siemens Prima 3.0T MRI scanner. Before and after intravenous (iv) injection of Gd-FLPNPs, the images including $\mathrm{T}_{1}$-WI and its pseudocolor images of the nude mouse were obtained. During the whole scan process, the mouse was anesthetized by isoflurane and maintained at normal temperature. The $\mathrm{T}_{1}$-weighted image parameters were as follows: repetition time $700 \mathrm{~ms}$; echo time $12 \mathrm{~ms}$; field of view $120 \times 120 \mathrm{~mm}$; matrix size $400 \times 400$; slice thickness $2.0 \mathrm{~mm}$; number of acquisitions 14 .

\section{Evaluation of cellular uptake by flow cytometry (FCM)}

The cellular uptake of the DOX-loaded NPs was confirmed by fluorescence microscopy (Epics XL). KB cells were incubated in 6 -well plates at a density of $4.0 \times 10^{5}$ cells/well in $4 \mathrm{~mL}$ of folic acid deficient 1640 medium containing 10\% FBS, and then cultured with Gd-FLPNPs or Gd-LPNPs at a final DOX concentration of $2 \mu \mathrm{g} / \mathrm{mL}$. At predetermined intervals, the cells were washed with PBS to remove the free NPs that did not enter the cells prior to fluorescence observation.

\section{In vitro cellular uptake by confocal laser scanning microscopy (CLSM)}

CLSM (Nikon TE2000; Nikon Corporation, Tokyo, Japan) was used to examine the intracellular distribution of DOX. KB cells were seeded on slides at a density of $5.0 \times 10^{4}$ cells/well in $1 \mathrm{~mL}$ of folic acid deficient 1640 medium containing $10 \%$ FBS. The cells were then incubated with Gd-FLPNPs or Gd-LPNPs at a final DOX concentration of $2 \mu \mathrm{g} / \mathrm{mL}$. At predetermined intervals, the cells were washed with PBS and fixed with 4\% (w/v) paraformaldehyde aqueous solution for 10 minutes. The slides were then stained with Hoechst $33258(5 \mathrm{mg} / \mathrm{mL}$ in PBS $)$ at $37^{\circ} \mathrm{C}$ for 10 minutes. The fixed cell monolayer was finally observed by CLSM.

\section{Endocytosis inhibition}

$\mathrm{KB}$ cells were seeded on slides on a 6-well plate at a density of $5.0 \times 10^{4}$ cells/well in $1 \mathrm{~mL}$ of folic acid deficient 1640 medium containing $10 \% \mathrm{FBS}$. Then, the cells were cultured for 1 day at $37^{\circ} \mathrm{C}$ in $5 \% \mathrm{CO}_{2}$ atmosphere. The cells were pretreated for 30 minutes with three different endocytosis inhibitors separately at concentrations of $5 \mathrm{mM}$ methyl-beta-cyclodextrin, $0.45 \mathrm{mM}$ sucrose, or $5 \mathrm{mM}$ cytochalasin D. Then, DOXloaded Gd-FLPNPs were added at a final DOX concentration of $2 \mu \mathrm{g} / \mathrm{mL}$, followed by incubation for 2 hours. The subsequent steps were similar to those described in the "In vivo $\mathrm{T}_{1}$-weighted MRI" section. The group with test materials but without inhibitor treatment was used as a control.

\section{In vitro cytotoxicity assay}

Cytotoxicity of Gd-FLPNPs or Gd-LPNPs was evaluated by 3-(4,5-dimethyl-2-thiazolyl)-2,5-diphenyl-2-H-tetrazolium bromide (MTT) assay. Briefly, KB cells were seeded on a 96-well plate (Costar, Cambridge, MA, USA) at a density of $5.0 \times 10^{3}$ cells/well in $100 \mu \mathrm{L}$ of folic acid deficient 1640 medium containing 10\% FBS. The cells were cultured for 24 hours at $37^{\circ} \mathrm{C}$ in $5 \% \mathrm{CO}_{2}$ atmosphere. Afterwards, the nonloaded nanocarrier, DOX-free Gd-FLPNPs, DOX-free Gd-LPNPs, DOX-loaded Gd-FLPNPs, DOX-loaded GdLPNPs, and free DOX at various concentrations, were added to each well. After incubation for 8 hours, the media were replaced by fresh folic acid deficient 1640 medium. After culture for 38 hours, MTT solution $(5 \mathrm{mg} / \mathrm{mL}$ in PBS, $20 \mu \mathrm{L}$ ) 
was added to each well and incubated for 4 hours. The media were removed and $150 \mu \mathrm{L}$ of DMSO was added to each well to dissolve the formazan blue crystal. The absorbance of the solution was measured using a microplate reader at $570 \mathrm{~nm}$. Cell viability was calculated as a percentage of the control (cells receiving no treatment).

\section{Antitumor assessment and histological analysis in vivo}

The sizes of the tumor in the mice models were measured every other day. When the tumor grew up to $\sim 50 \mathrm{~cm}^{3}$, 20 mice were divided into four groups and intravenously injected with PBS, free DOX, Gd-LPNPs, and Gd-FLPNPs. Tumor volume was estimated by the following equation: $\mathrm{V}=\mathrm{a} \times \mathrm{b}^{2} / 2$, where $\mathrm{a}$ and $\mathrm{b}$ were the longest and shortest diameters, respectively.

\section{Results}

\section{Preparation of Gd-FLPNPs}

The synthesis process of Gd-DTPA-BC ${ }_{16}$ and mPEG-S-S-C are presented in Figures 2A and S1. The carboxyl groups of DTPA were combined with the amino groups of cetylamine.
Then, $\mathrm{Gd}^{3+}$ was incorporated into DTPA-BC $\mathrm{B}_{16}$. The structures of Gd-DTPA-BC ${ }_{16}$ and mPEG-S-S-C ${ }_{16}$ were identified by ${ }^{1} \mathrm{H}$ NMR spectra as shown in Figures $2 \mathrm{~B}$ and $\mathrm{S} 2$, and by infrared spectroscopy (Figure 2C). After that, Gd-FLPNPs were prepared using a reported self-assembly method. ${ }^{25}$ Gd-FLPNPs were made up of two components (Figure 1): a hydrophobic PLGA core, and a paramagnetic and redoxsensitive liposome shell. The elements on the core-shell Gd-FLPNPs surface were identified according to the specific binding energy of $\mathrm{Gd}^{4 \mathrm{~d}}$ using XPS (Figure 3D and E), which indicated the successful coating of Gd-DTPA-BC ${ }_{16}$ on the NPs. As shown in Figure 3A, a clear core-shell structure is visible from the TEM images. The images also show that Gd-FLPNPs were dispersed individually with a well-defined spherical shape and a smooth surface. The paramagnetic lipid shell about 10-20 nm was fused onto the hydrophobic PLGA core (Figure 3A). The merit of this structure is that poorly soluble drugs can be encapsulated into the hydrophobic PLGA cavity with a high LE. At the same time, the multifunctional liposome shell offers many advantages such as folate targeting, redox-sensitivity, and contrast-enhanced ability for diagnosis and therapy.
A

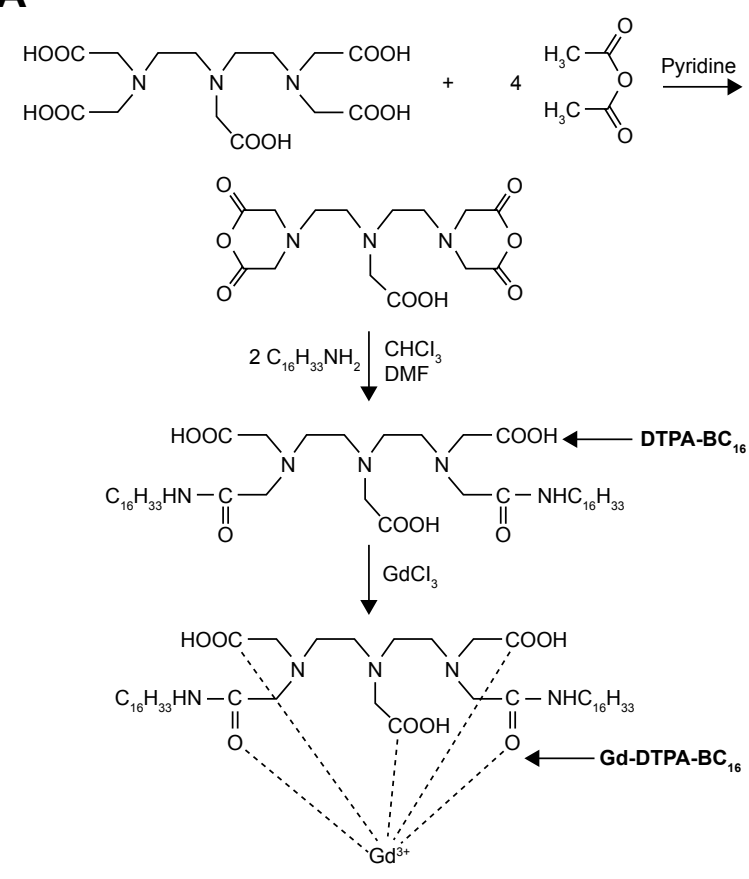

B

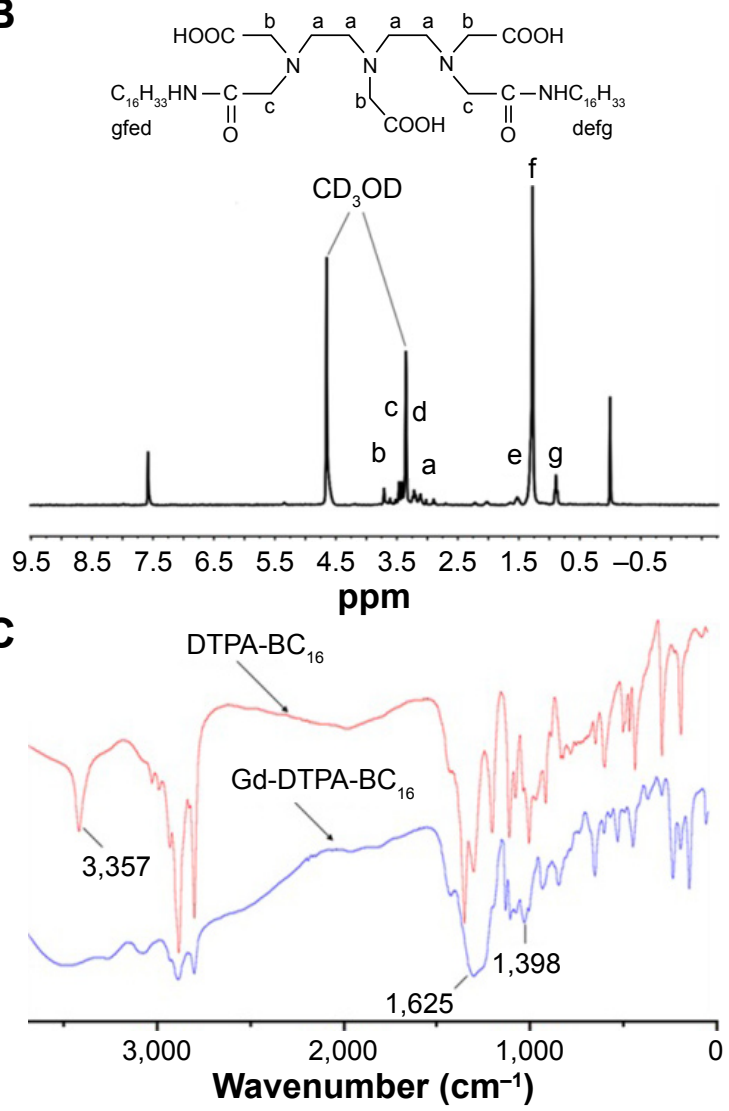

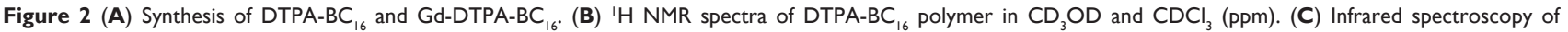
Gd-DTPA-BC 16 and DTPA-BC 16 .

Abbreviations: DMP, N,N-dimethylformamide; Gd-DTPA-BC ${ }_{16}$, gadolinium-diethylenetriaminepentaacetic acid-bis-tetradecylamide; NMR, nuclear magnetic resonance. 
A

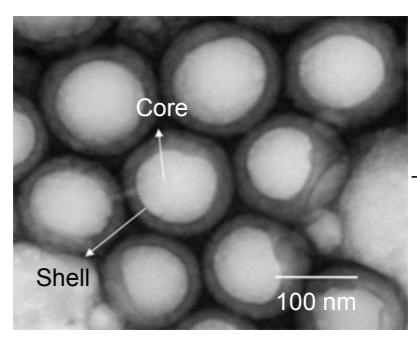

B
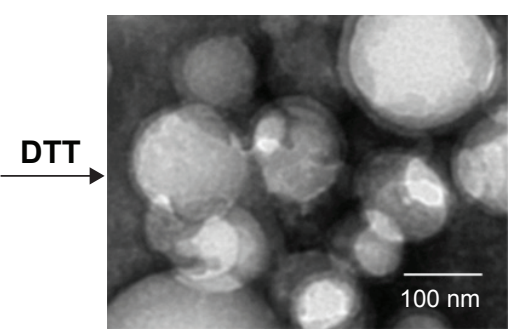

D

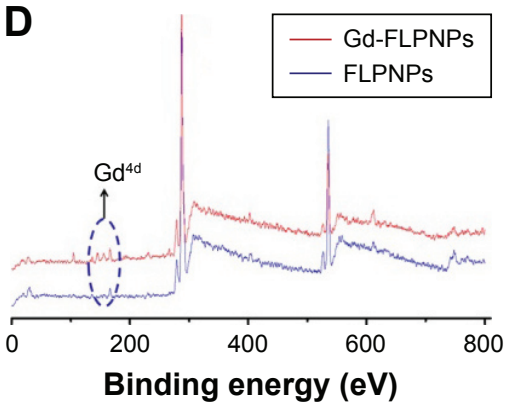

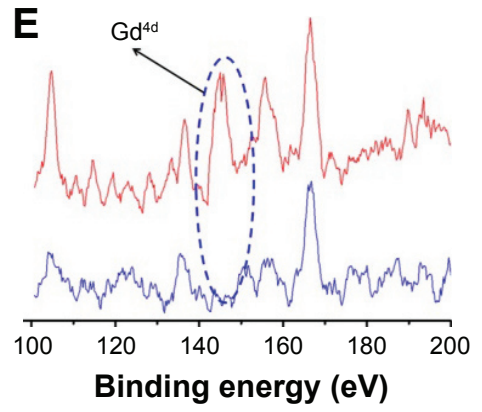

C
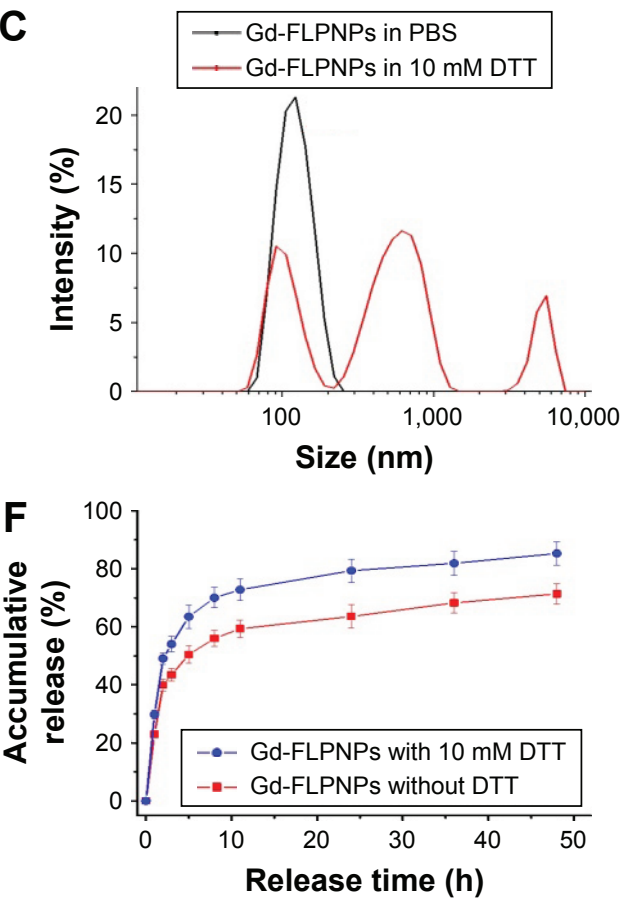

Figure 3 Characterizations of Gd-FLPNPs.

Notes: (A) TEM image of Gd-FLPNPs. (B) TEM image of Gd-FLPNPs treated with DTT (10 mM) for 4 hours. (C) Size change of Gd-FLPNPs in response to I0 mM DTT in PBS after 4 hours (0.0I M, pH =7.4) determined by DLS. (D) XPS spectra of the samples. (E) Gd $\mathrm{d}^{4 \mathrm{~d}}$ signal spectra of Gd-FLPNPs. (F) Redox-triggered release of DOX from Gd-FLPNPs in PBS (0.0I M, pH 7.4) with or without $10 \mathrm{mM}$ DTT.

Abbreviations: Gd-FLPNPs, Gd-coated folate-targeted lipid-polymer hybrid nanoparticles; DOX, doxorubicin; TEM, transmission electron microscopy; DTT, DLdithiothreitol; PBS, phosphate-buffered saline; DLS, dynamic light scattering; XPS, X-ray photoelectron spectroscopy.

\section{Characterization of Gd-FLPNPs}

The size and size distribution of Gd-FLPNPs were characterized by DLS (Table 1; Figure 3C). The average size of Gd-FLPNPs was about $120-130 \mathrm{~nm}$, which is conducive to satisfactory drug accumulation in the tumor site through the enhanced permeability and retention (EPR) effect. ${ }^{26,27}$ The polydispersity of Gd-FLPNPs was 0.106 , which indicated a unimodal size distribution. In this study, DOX was successfully loaded into Gd-FLPNPs with a satisfying loading efficiency (LE) and encapsulation efficiency (EE); the LE and EE of Gd-FLPNPs were about $5.4 \%$ and $76.3 \%$, respectively (Table 1).

As a redox-sensitive NP, Gd-FLPNPs exhibit a rapid response to reducing environments (Figure 3B). The change

Table I Physicochemical characterization of Gd-FLPNPs and Gd-LPNPs

\begin{tabular}{lllll}
\hline Sample & $\begin{array}{l}\text { Particle } \\
\text { size }(\mathbf{n m})\end{array}$ & PDI & EE (\%) & LE (\%) \\
\hline Gd-FLPNPs & $122.6 \pm 5.2$ & 0.106 & 76.3 & 5.4 \\
Gd-LPNPs & $127.3 \pm 4.7$ & 0.154 & 74.2 & 5.3 \\
\hline
\end{tabular}

Note: Data represent mean $\pm S D, n=3$.

Abbreviations: Gd-FLPNPs, Gd-coated folate-targeted lipid-polymer hybrid nanoparticles; Gd-LPNPs, Gd-coated lipid-polymer hybrid nanoparticles; SD, standard deviation; PDI, polydispersity index; EE, encapsulation efficiency; LE, loading efficiency. of size distribution of Gd-FLPNPs in response to $10 \mathrm{mM} \mathrm{DTT}$ was monitored by TEM (Figure 3B) and DLS (Figure 3C). Due to the cleavage of the disulfide linkage, the size of GdFLPNPs significantly increased after 4-hour co-incubation of DTT, indicating the falling off of the hydrophilic PEG shell from the Gd-FLPNPs. Destabilization and aggregation of the hydrophobic inner core in the reducing environments are conducive to the release of drug and CAs in the tumor tissue, and result in tumor-specific enhanced imaging and therapy.

\section{In vitro redox-sensitivity release}

The in vitro redox-sensitive release behavior of Gd-FLPNPs was further analyzed using a dialysis method. As shown in Figure 3, the Gd-FLPNPs incubated with DTT showed more efficient and fast release of DOX from Gd-FLPNPs in the same period. In contrast, less DOX was released in the absence of DTT. For Gd-FLPNPs, about $79.3 \%$ of the payload was released over a period of 24 hours in the presence of $10 \mathrm{mM}$ DTT, while $63.4 \%$ of the payload was released in the absence of DTT. This result may be related to the cleavage of the disulfide bond of $\mathrm{mPEG}-\mathrm{S}-\mathrm{S}-\mathrm{C}_{16}$ in the reducing environment, which resulted in the dissociation of the NPs structure. These results suggested that the Gd-FLPNPs could 
release loading responsively, and may be a potential intracellular environment-sensitive drug delivery system.

\section{Targeted cell uptake and profile}

The targeted cellular uptake profiles of Gd-FLPNPs were examined with folate overexpressing KB cells (human oral epidermoid carcinoma cell line) using FCM. With the absence of folate in the culture medium, the KB cells with Gd-FLPNPs resulted in stronger DOX fluorescence than with Gd-LPNPs, in both the cytoplasm and nuclei. The mean DOX fluorescence value was 28.2 after 4 hours of incubation with Gd-FLPNPs in a folate-free medium, while it was 19.6 after incubation with non-targeted lipid-polymer hybrid nanoparticles (Gd-LPNPs) (Figure 4). We also examined the effects of the addition of folate into the medium. When folate-free medium was replaced with folate-contained medium, there was little difference in the cellular uptake of DOX between Gd-FLPNPs and Gd-LPNPs. As a result of the competitive binding to FRs, the folate in the culture medium prevented Gd-FLPNPs from transporting into KB cells. The internalization pathway of Gd-LPNPs was also evaluated using three types of endocytosis inhibitors. As shown in Figure 4B, the mean DOX fluorescence intensity decreased to $43 \%$ after treatment with hypertonic sucrose, which indicated that the pathway of Gd-FLPNPs into cells depended on clathrinmediated endocytosis. ${ }^{28}$

Cellular uptakes of Gd-FLPNPs and Gd-LPNPs by KB cells were further studied by CLSM. The results show that Gd-FLPNPs produced stronger DOX fluorescence than Gd-LPNPs after 4 hours of incubation (Figure 5). Moreover, when $\mathrm{KB}$ cells were incubated in the folate medium, Gd-FLPNPs showed a similar extent of cellular uptake as
Gd-LPNPs. This result suggested that the folate on the surface of NPs facilitated the entry of Gd-FLPNPs into cells, which is consistent with FCM results. These results fully proved that Gd-FLPNPs were transported to cells through an FRsmediated endocytosis process.

To further study the targeting efficacy of Gd-FLPNPs, KB cells were incubated with folate-targeted and non-targeted paramagnetic NPs. Figure 6C shows the Gd concentration, which was measured from the lysate of $\mathrm{KB}$ cells incubated with Magnevist, non-targeted Gd-LPNPs, and folate-targeted Gd-FLPNPs. Clearly, $\mathrm{Gd}^{3+}$ concentrations were higher after incubation with both types of NPs than with Magnevist. In addition, the uptake of Gd-FLPNPs $(0.24 \mathrm{mg} / \mathrm{L})$ was much more efficient than Gd-LPNPs $(0.15 \mathrm{mg} / \mathrm{L})$ by KB cells with an FRs-mediated endocytosis process. This means that Gd-LPNPs could provide high delivery efficiency of CAs and acquire higher contrast MRI.

\section{In vitro MRI}

Proton longitudinal relaxivity $\left(\mathrm{r}_{1}\right)$, which is an important parameter revealing the ability of a CA for MRI images, was calculated from the change of water proton relaxation rate $1 / \mathrm{T}_{1}\left(\mathrm{~s}^{-1}\right)$ per $\mathrm{mM}$ concentration. To obtain the $\mathrm{T}_{1}$-weighted MRI images and $r_{1}$, solutions containing different concentrations of Gd-FLPNPs or Magnevist were placed in a 96-well plate and tested by a Siemens Prima 3.0T MRI scanner. $\mathrm{T}_{1}$-weighted MRI images of the phantom are shown in Figure 6A. The signal intensity of Gd-FLPNPs was clearly better than that of Magnevist. After fitting, the $r_{1}$ of GdFLPNPs was $8.02 \mathrm{mM}^{-1} \mathrm{~s}^{-1}$ as shown in Figure 6B. In contrast, the $r_{1}$ value of small molecule Magnevist was $4.61 \mathrm{mM}^{-1} \mathrm{~s}^{-1}$. The results indicate that Gd-FLPNPs hold better contrast
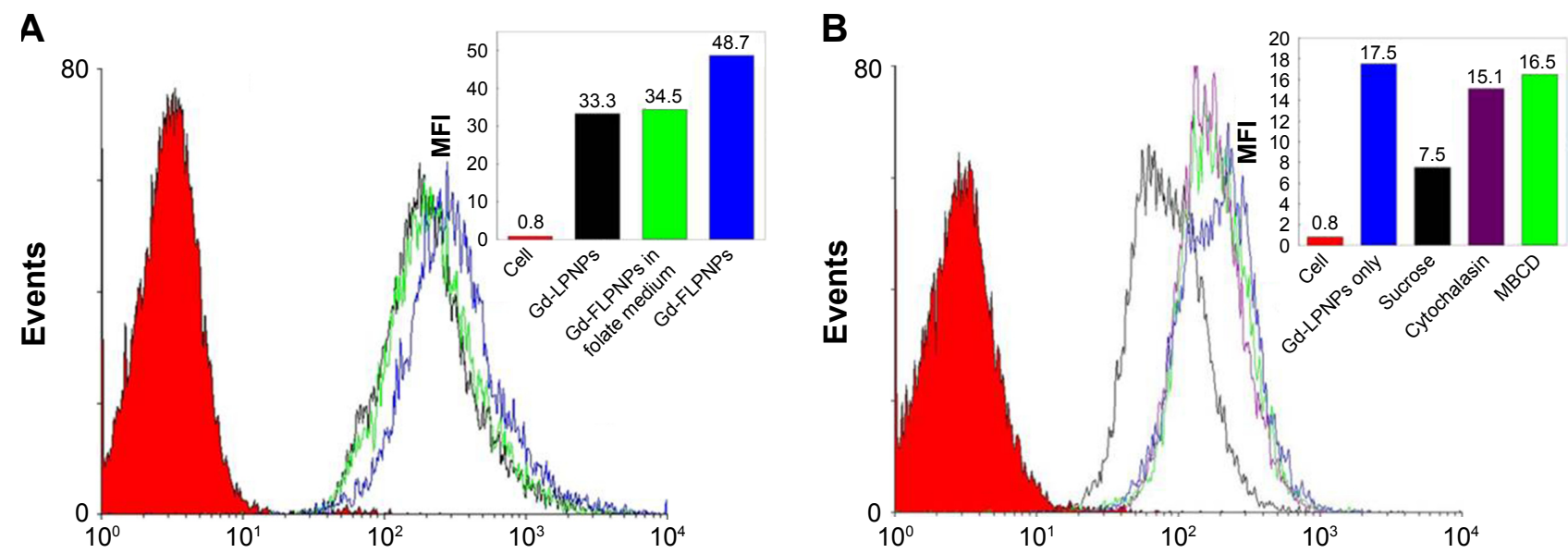

Figure 4 Study of targeted cell uptake and internalization pathway by FCM.

Notes: (A) Quantitative flow cytometry analysis of the cellular DOX red fluorescence MFI values of KB cells incubated with Gd-FLPNPs for I0 hours; DOX dosage was $2 \mu \mathrm{g} / \mathrm{mL}$. (B) Effect of endocytosis inhibitors on the uptake of Gd-FLPNPs in KB cells using flow cytometry analyses; DOX dosage was $2 \mu \mathrm{g} / \mathrm{mL}$.

Abbreviations: FCM, flow cytometry; Gd-FLPNPs, Gd-coated folate-targeted lipid-polymer hybrid nanoparticles; DOX, doxorubicin; Gd-LPNPs, Gd-coated lipid-polymer hybrid nanoparticles; MBCD, methyl-beta-cyclodextrin; MFI, mean fluorescence intensity of cellular DOX red fluorescence. 

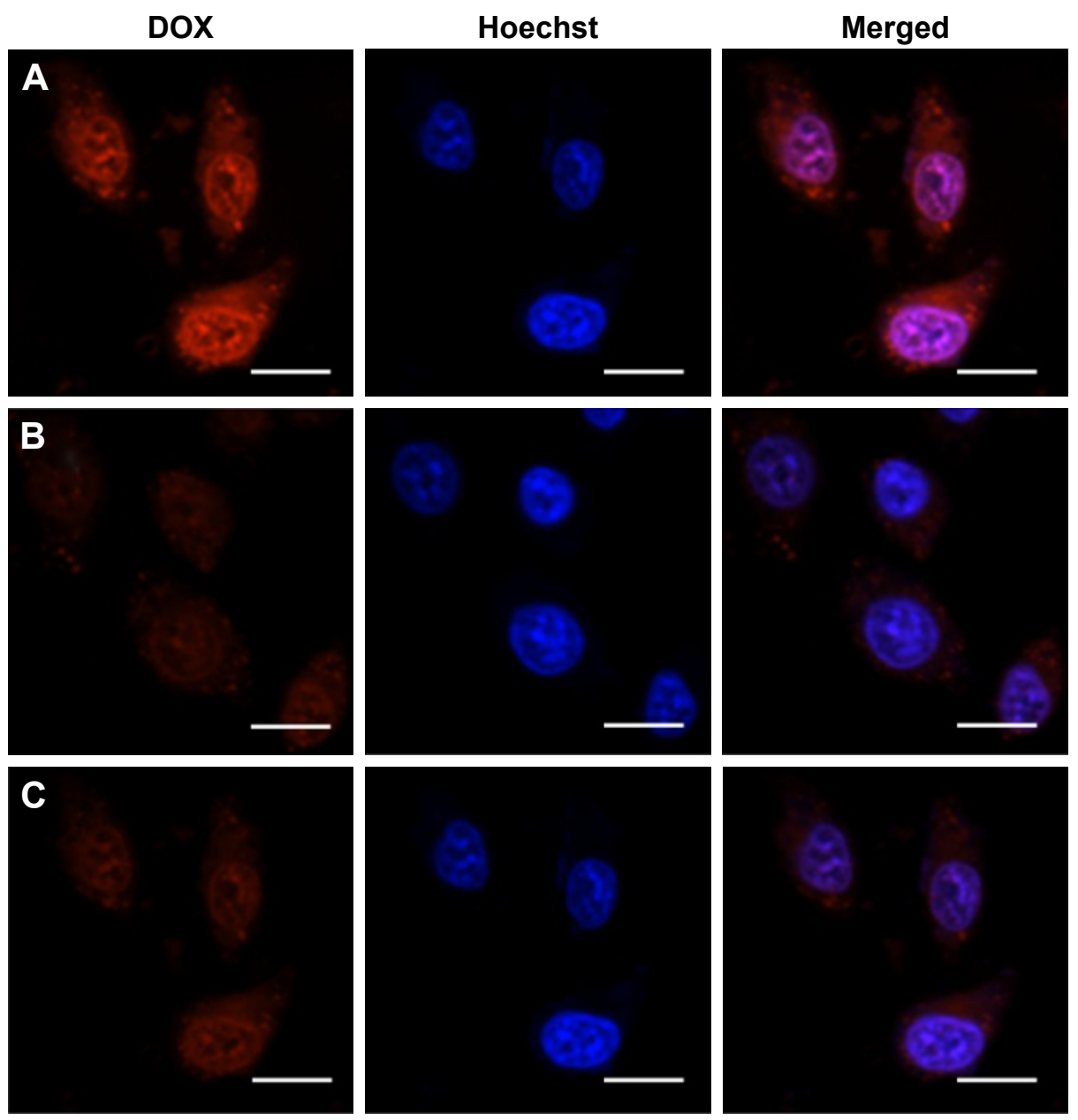

Figure 5 CLSM images of KB cells incubated with (A) Gd-FLPNPs, (B) Gd-LPNPs, and (C) Gd-FLPNPs pretreated with excess of folate acid for 4 hours. Note: DOX: red fluorescence images; nuclei: blue fluorescence images; blue and red fluorescence: merged images (scale bar, $20 \mu \mathrm{m})$.

Abbreviations: Gd-FLPNPs, Gd-coated folate-targeted lipid-polymer hybrid nanoparticles; DOX, doxorubicin; Gd-LPNPs, Gd-coated lipid-polymer hybrid nanoparticles; CLSM, confocal laser scanning microscopy.

capability than Magnevist at the same concentration. As reported previously, the proton relaxivity of lanthanide ions in water could be influenced by a combination of factors, that may be the reason of improvement of macromolecular CAs. ${ }^{29,30}$ It has been demonstrated that due to a prolongation of the rotational correlation time $(\tau R)$, relaxivity improves with the incorporation of paramagnetic ions into a macromolecular structure. ${ }^{31,32}$

\section{In vivo $M R I$}

To study the MRI contrast effect of Gd-FLPNPs, $\mathrm{T}_{1}$-weighted $\mathrm{MR}$ images were acquired using nude mice bearing C6 tumor xenografts after injection of Gd-FLPNPs at different time points. Compared with the image of preinjection, an obvious enhanced effect was found in $\mathrm{T}_{1}$-weighted MR image of the tumor as shown in Figure 6D. Obvious $T_{1}$ hyperintense signals could be found after 1 hour injection of Gd-FLPNPs, and the contrast enhancement in
$\mathrm{T}_{1}$-weighted $\mathrm{MR}$ image reached a maximum 4 hours after injection. Then, the signal intensity decreased gradually with the time prolonging. Further quantitative analysis showed that MR signals revealed $T_{1}$ signal intensity enhancement by $36 \%$, suggesting high tumor accumulation of the NPs after systemic administration. Additionally, due to the EPR effect of NPs, Gd-FLPNPs provided a long time window to obtain high-quality MRI for guidance and to monitor the effect of chemotherapy compared to commercial CA (Magnevist).

\section{Antitumor effects of Gd-FLPNPs in vitro and in vivo}

The antitumor effects of Gd-FLPNPs, Gd-LPNPs, and free DOX against KB cells were evaluated by the MTT assay. Before that, the KB cells were incubated with different concentrations of DOX-free Gd-FLPNPs, DOX-free Gd-LPNPs, or Magnevist. The result proves that neither the 
A

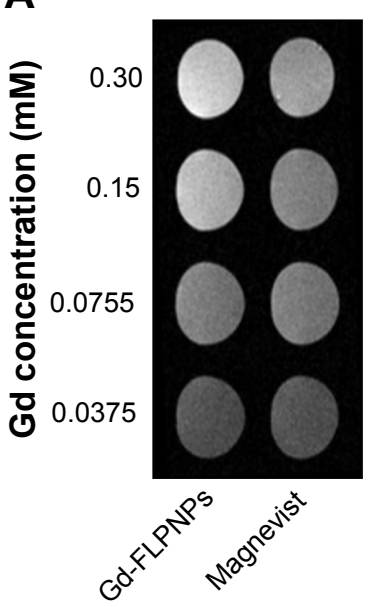

B

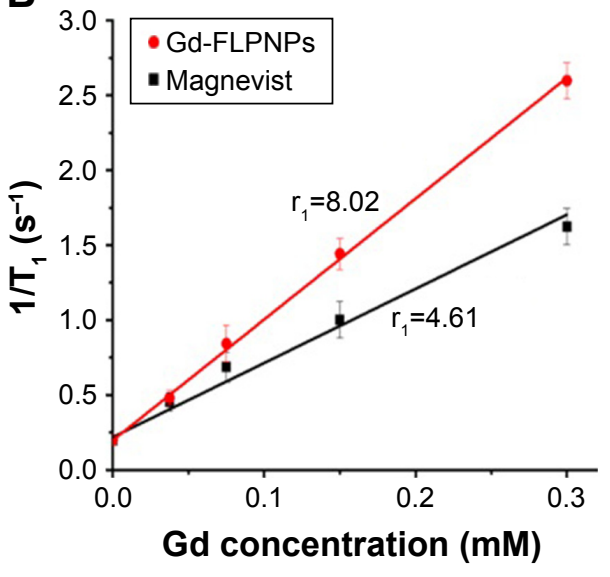

C

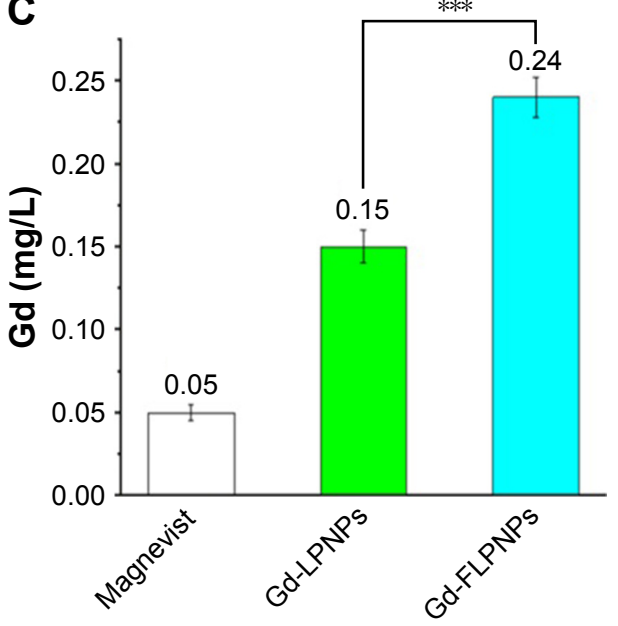

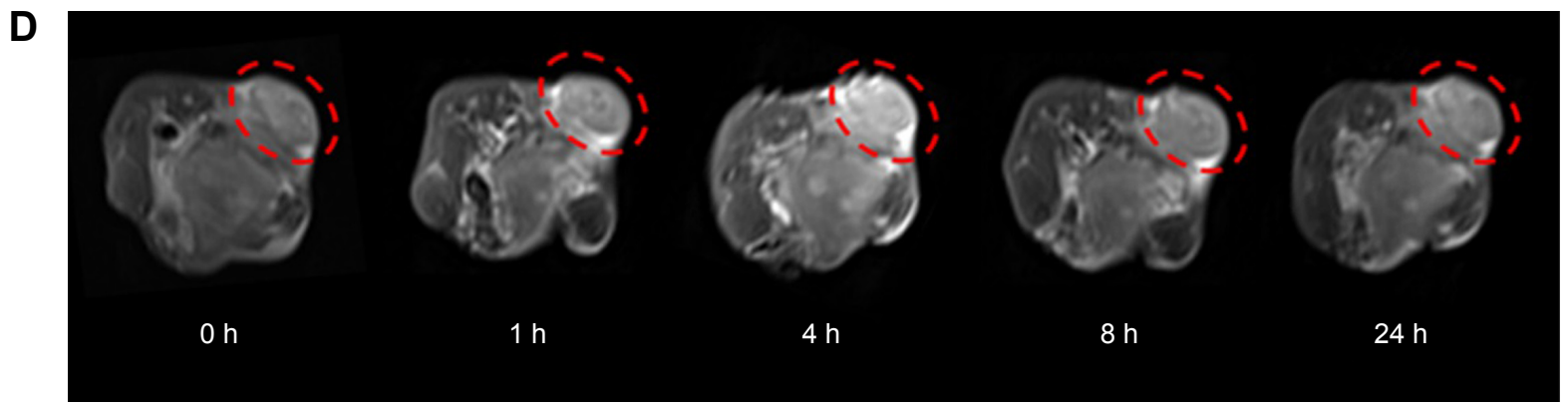

Figure 6 The contrast effect of Gd-FLPNPs.

Notes: (A) Phantom MR images of Gd-FLPNPs in comparison to Magnevist showing T,-weighted bright contrast. (B) Relaxivity plot for Gd-FLPNPs and Magnevist. (C) ICPAES measurements of cellular Gd concentration with I0 hours post-uptake of Gd-FLPNPs $* * *(P<0.00 \mathrm{I})$. (D) In vivo MR images of human oral epidermoid carcinoma-bearing mice pre- and post-intravenous injection of Gd-FLPNPs. The red circled area marks the tumor location.

Abbreviations: MR, magnetic resonance; Gd-FLPNPs, Gd-coated folate-targeted lipid-polymer hybrid nanoparticles; Gd-LPNPs, Gd-coated lipid-polymer hybrid nanoparticles; ICP-AES, inductively coupled plasma atomic emission spectroscopy; DTPA, diethylenetriaminepentaacetic acid.

NPs nor Magnevist has a negligible effect on the survival rate of $\mathrm{KB}$ cells at any concentration (Figure 7A). As shown in Figure 7B, all DOX-loaded materials notably inhibited the growth of KB cells. The inhibitory effects on the cell viability were dose-dependent. The $\mathrm{IC}_{50}$ of Gd-FLPNPs, Gd-LPNPs, and free DOX were about $0.98,1.75$, and $0.34 \mu \mathrm{g} / \mathrm{mL}$, respectively. Since small molecules can be more easily transported into cells and nuclei by passive diffusion, free DOX was more cytotoxic to KB cells than the NPs as shown in Figure 7B. Predictably, at the same dose of DOX, the cytotoxicity of Gd-FLPNPs was significantly higher than that of Gd-LPNPs, which was consistent with the results observed by CLSM and FCM. Moreover, with $2 \mathrm{mM}$ folate in the medium, the cytotoxicity of Gd-FLPNPs was noticeably reduced and became nearly equivalent to that of Gd-LPNPs. This fact indicated that the cell uptake of Gd-FLPNPs was an FRs-mediated endocytosis process.

The in vivo antitumor effect of Gd-FLPNPs was evaluated using nude mice bearing $\mathrm{KB}$ tumor xenografts, and the tumor volumes of the mice were monitored every other day. In the test group, mice were caudal vein injected with
PBS solution of Gd-FLPNPs and Gd-LPNPs. As shown in Figure $7 \mathrm{C}$ and D, the mice inoculated tumors with PBS only grew rapidly. On day 14 , the mean tumor volume was about nine times as big as the initial volume. Free DOX group showed antitumor effect to some extent against the subcutaneous tumor in mice, especially in the early stage of tumor, and then the tumor continued to increase in size. In contrast, after treating with Gd-FLPNPs, tumor growth was inhibited by $64 \%$, while in the group of Gd-LPNPs it was inhibited by $45 \%$. Compared with the non-targeted group, the folate active targeting ligand enables sufficient accumulation of Gd-FLPNPs in tumor tissues, and then showed significant antitumor effects. The above results confirmed the potential folate-targeted antitumor effect of Gd-FLPNPs.

The potential in vivo toxicity induced by different treatments was evaluated by the body weight of animals after treatment. There was no big difference in body weight loss after treatments for up to 14 days in the test groups, demonstrating that all treatments including iv injection of the agent and drugs had good biocompatibility and biosafety. 

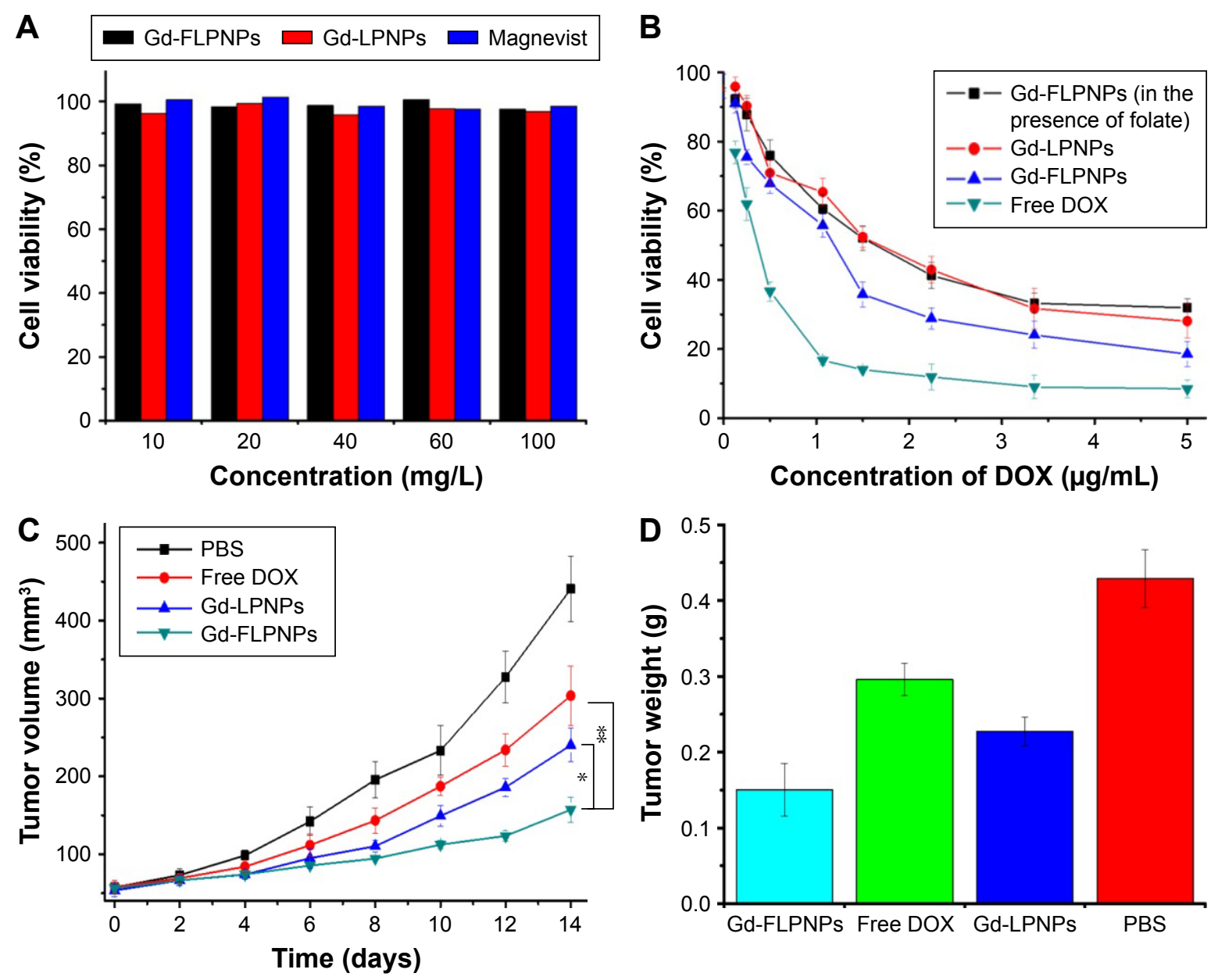

Figure 7 In vitro and in vivo cancer therapy in $\mathrm{KB}$ tumor-bearing mice.

Notes: (A) Cell viability of KB cells incubated with non-DOX loaded Gd-FLPNPs for 48 hours by MTT assay. (B) Cytotoxicity of Gd-FLPNPs, Gd-LPNPs, Gd-FLPNPs pretreated with excess of folate $(0.2 \mathrm{mM}$ free folate acid was added to the medium), and free DOX against KB cells after incubation for 8 hours ( $\mathrm{n}=4)$. (C) Changes in the relative tumor volume $(\mathrm{n}=4)$ after intravenous injection at days 0,5 , and $10(\mathrm{DOX}$ dosage: $6 \mathrm{mg} / \mathrm{kg})$. $*(P<0 . \mathrm{I})$ and $* *(P<0.0 \mathrm{I})$. (D) Relative tumor weight of the mice with the various treatments.

Abbreviations: Gd-FLPNPs, Gd-coated folate-targeted lipid-polymer hybrid nanoparticles; DOX, doxorubicin; Gd-LPNPs, Gd-coated lipid-polymer hybrid nanoparticles; MTT, 3-(4,5-dimethyl-2-thiazolyl)-2,5-diphenyl-2-H-tetrazolium bromide; PBS, phosphate-buffered saline.

\section{Conclusion}

In summary, we developed a multifunctional NP system where the targeting ligand, CA, and anticancer drugs could all be incorporated into one platform. We demonstrated that Gd-FLPNPs have high monodispersity, excellent size stability, and a well-defined core-shell structure. In vitro release experiments showed that Gd-FLPNPs as a reduction-sensitive drug deliver released DOX faster with the presence of DTT than without it. In vitro and in vivo MRI images showed that this type of NP had paramagnetic properties with about two-fold enhancement in the longitudinal relaxivity compared to the clinically used Magnevist. That confirmed the potential of Gd-FLPNPs as an effective $\mathrm{T}_{1}$-WI MRI CA. Furthermore, FCM, confocal image, and MTT assay revealed that Gd-FLPNPs further enhanced cell uptake and produced higher cytotoxicity against KB cells than non-targeted Gd-LPNPs, which can reduce side effects of anticancer drugs at a desired location. In conclusion, the multifunctional drug delivery system is a promising platform for image-guided drug delivery systems.

\section{Acknowledgments}

We thank Ping Yu for technical assistance, Lei Liu for the illustrations and digital pictures, and Liu-Jie Zhang for cell experiments. The authors acknowledge funding from the National Natural Science Foundation of China (81372369, 51473127, 81571734 and 51673152), China Postdoctoral Science Foundation (2017M612514), and Zhongnan Hospital of Wuhan University Science, Technology and Innovation Seed Fund Project (cxpy20160011).

\section{Disclosure}

The authors report no conflicts of interest in this work. 


\section{References}

1. Weissleder R, Pittet MJ. Imaging in the era of molecular oncology. Nature. 2008;452(7187):580-589.

2. Mi P, Cabral H, Kokuryo D, et al. Gd-DTPA-loaded polymer-metal complex micelles with high relaxivity for MR cancer imaging. Biomaterials. 2013;34(2):492-500.

3. Hubbell JA, Chilkoti A. Nanomaterials for drug delivery. Science. 2012; 337(6092):303-305.

4. Xu W, Burke JF, Pilla S, Chen H, Jaskula-Sztul R, Gong S. Octreotidefunctionalized and resveratrol-loaded unimolecular micelles for targeted neuroendocrine cancer therapy. Nanoscale. 2013;5(20):9924-9933.

5. Floyd WC, Klemm PJ, Smiles DE, et al. Conjugation effects of various linkers on Gd(III) MRI contrast agents with dendrimers: optimizing the hydroxypyridinonate (HOPO) ligands with nontoxic, degradable esteramide (EA) dendrimers for high relaxivity. J Am Chem Soc. 2011; 133(8):2390-2393.

6. Li X, Zhou H, Yang L, et al. Enhancement of cell recognition in vitro by dual-ligand cancer targeting gold nanoparticles. Biomaterials. 2011; 32(10):2540-2545.

7. Cheng ZL, Tsourkas A. Monitoring phospholipase $A_{2}$ activity with Gd-encapsulated phospholipid liposomes. Sci Rep. 2014;4:6958.

8. Li XY, Wu MY, Pan LM, Shi JL. Tumor vascular-targeted co-delivery of anti-angiogenesis and chemotherapeutic agents by mesoporous silica nanoparticle-based drug delivery system for synergetic therapy of tumor. Int J Nanomedicine. 2016;11:93-105.

9. Li L, ten Hagen TLM, Haeri A, et al. A novel two-step mild hyperthermia for advanced liposomal chemotherapy. J Control Release. 2014; 174:202-208.

10. Wang S, Wang H, Liu Z, et al. Smart pH- and reduction-dual-responsive folate-PEG-coated polymeric lipid vesicles for tumor-triggered targeted drug delivery. Nanoscale. 2014;6(13):7635-7642.

11. Karimi M, Ghasemi A, Sahandi Zangabad P, et al. Smart micro/ nanoparticles in stimulus-responsive drug/gene delivery systems. Chem Soc Rev. 2016;45(5):1457-1501.

12. El-Dakdouki MH, El-Boubbou K, Kamat M, et al. CD44 targeting magnetic glyconanoparticles for atherosclerotic plaque imaging. Pharm Res. 2014;31(6):1426-1437.

13. Wu B, Yu P, Cui C, et al. Folate-containing reduction-sensitive lipidpolymer hybrid nanoparticles for targeted delivery of doxorubicin. Biomater Sci. 2015;3(4):655-664.

14. Cui C, Xue YN, Wu M, et al. Cellular uptake, intracellular trafficking, and antitumor efficacy of doxorubicin-loaded reduction-sensitive micelles. Biomaterials. 2013;34(15):3858-3869.

15. Zhang LJ, Wu B, Zhou W, et al. Two-component reduction-sensitive lipid-polymer hybrid nanoparticles for triggered drug release and enhanced in vitro and in vivo anti-tumor efficacy. Biomater Sci. 2016;5(1): 98-110.

16. Chen W, Zhong P, Meng F, et al. Redox and pH-responsive degradable micelles for dually activated intracellular anticancer drug release. J Control Release. 2013;169(3):171-179.
17. Singh R, Karakoti AS, Self W, Seal S, Singh S. Redox-sensitive cerium oxide nanoparticles protect human keratinocytes from oxidative stress induced by glutathione depletion. Langmuir. 2016;32(46): 12202-12211.

18. Mura S, Nicolas J, Couvreur P. Stimuli-responsive nanocarriers for drug delivery. Nat Mater. 2013;12(11):991-1003.

19. Wu B, Lu ST, Zhang LJ, Zhuo RX, Xu HB, Huang SW. Codelivery of doxorubicin and triptolide with reduction-sensitive lipid-polymer hybrid nanoparticles for in vitro and in vivo synergistic cancer treatment. Int $J$ Nanomedicine. 2017;12:1853-1862.

20. Vergote IB, Marth C, Coleman RL. Role of the folate receptor in ovarian cancer treatment: evidence, mechanism, and clinical implications. Cancer Metastasis Rev. 2015;34(1):41-52.

21. Satsangi A, Roy SS, Satsangi RK, et al. Synthesis of a novel, sequentially active-targeted drug delivery nanoplatform for breast cancer therapy. Biomaterials. 2015;59:88-101.

22. Shi D. Integrated multifunctional nanosystems for medical diagnosis and treatment. Adv Funct Mater. 2009;19(21):3356-3373.

23. Pan J, Feng SS. Targeting and imaging cancer cells by folate-decorated, quantum dots (QDs)-loaded nanoparticles of biodegradable polymers. Biomaterials. 2009;30(6):1176-1183.

24. Kimpe K, Parac-Vogt Tatjana N, Laurent S, et al. Potential MRI contrast agents based on micellar incorporation of amphiphilic bis(alkylamide) derivatives of $\left[(\mathrm{Gd}-\mathrm{DTPA})\left(\mathrm{H}_{2} \mathrm{O}\right)\right]^{2-}$. Eur J Inorg Chem. 2003;16:3021-3027.

25. Taratula O, Kuzmov A, Shah M, Garbuzenko OB, Minko T. Nanostructured lipid carriers as multifunctional nanomedicine platform for pulmonary co-delivery of anticancer drugs and siRNA. J Control Release. 2013;171(3):349-357.

26. Maeda H, Wu J, Sawa T, Matsumura Y, Hori K. Tumor vascular permeability and the EPR effect in macromolecular therapeutics: a review. $J$ Control Release. 2000;65(1):271-284.

27. Fang J, Nakamura H, Maeda $H$. The EPR effect: unique features of tumor blood vessels for drug delivery, factors involved, and limitations and augmentation of the effect. Adv Drug Deliv Rev. 2011;63(3): 136-151.

28. Oh JM, Choi SJ, Kim ST, Choy JH. Cellular uptake mechanism of an inorganic nanovehicle and its drug conjugates: enhanced efficacy due to clathrin-mediated endocytosis. Bioconjug Chem. 2006;17(6): 1411-1417.

29. Solomon I. Relaxation processes in a system of two spins. Phys Rev. 1955; 99(2):559-565.

30. Jung SH, Na K, Lee SA, Cho SH, Seong H, Shin BC. Gd(III)-DOTAmodified sonosensitive liposomes for ultrasound-triggered release and MR imaging. Nanoscale Res Lett. 2012;7(1):462.

31. Hak S, Sanders HMHF, Agrawal P, et al. A high relaxivity Gd(III) DOTA-DSPE-based liposomal contrast agent for magnetic resonance imaging. Eur J Pharm Biopharm. 2009;72(2):397-404.

32. Kielar F, Tei L, Terreno E, Botta M. Large relaxivity enhancement of paramagnetic lipid nanoparticles by restricting the local motions of the GdIII chelates. J Am Chem Soc. 2010;132(23):7836-7837. 


\section{Supplementary materials}

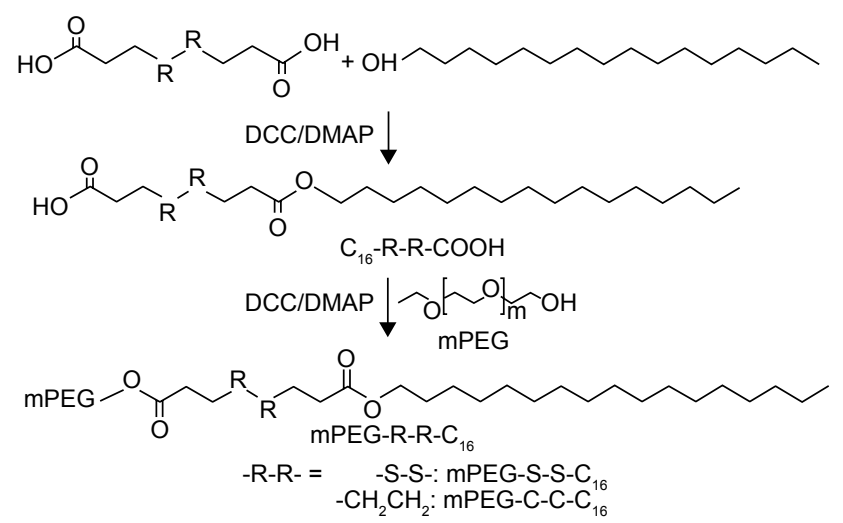

Figure SI Synthesis pathway of the amphiphilic polymers (mPEG-S-S- $\mathrm{C}_{16}$ ).

Abbreviations: DCC, dicyclohexylcarbodiimide; DMAP, 4-dimethylaminopyridine; mPEG-S-S-C ${ }_{16}$, monomethoxy-poly-(ethylene glycol)-S-S-hexadecyl.

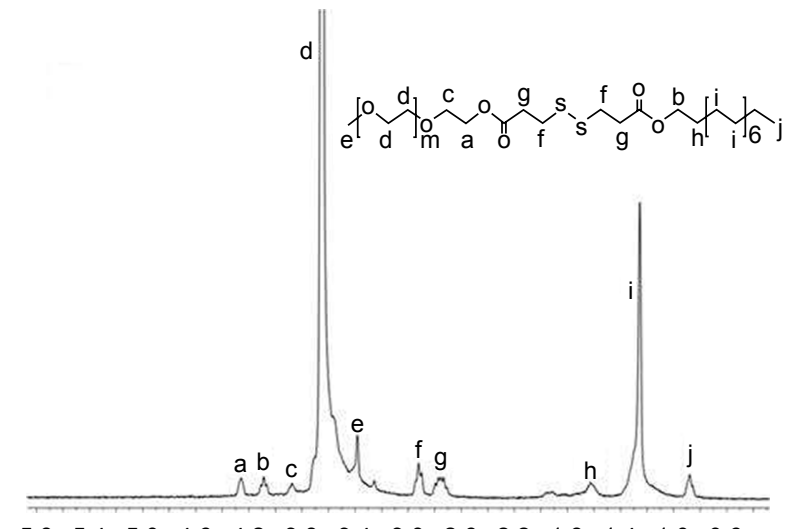

$\begin{array}{llllllllllllll}5.8 & 5.4 & 5.0 & 4.6 & 4.2 & 3.8 & 3.4 & 3.0 & 2.6 & 2.2 & 1.8 & 1.4 & 1.0 & 0.6\end{array}$

Figure S2 'H NMR spectra of mPEG-S-S- $\mathrm{C}_{16}$ polymer in $\mathrm{CDCl}_{3}$ (ppm). Abbreviations: mPEG-S-S-C ${ }_{16}$, monomethoxy-poly-(ethyleneglycol)-S-S-hexadecyl; NMR, nuclear magnetic resonance.

\section{Publish your work in this journal}

The International Journal of Nanomedicine is an international, peerreviewed journal focusing on the application of nanotechnology in diagnostics, therapeutics, and drug delivery systems throughout the biomedical field. This journal is indexed on PubMed Central, MedLine, CAS, SciSearch $®$, Current Contents ${ }^{\circledR} /$ Clinical Medicine,
Journal Citation Reports/Science Edition, EMBase, Scopus and the Elsevier Bibliographic databases. The manuscript management system is completely online and includes a very quick and fair peer-review system, which is all easy to use. Visit http://www.dovepress.com/ testimonials.php to read real quotes from published authors. 